\title{
HTS Current Leads: Performance Overview in Different Operating Modes
}

\author{
A. Ballarino
}

\begin{abstract}
High Temperature Superconducting (HTS) current leads have become, in the last few years, a valid choice for the powering of superconducting magnet systems. After the construction and test of more than a thousand of HTS leads for the Large Hadron Collider, with a consequent improved confidence in the long-term and large scale operation of these devices, it is being envisaged to equip several other large superconducting magnet systems with this type of lead. The approach used in the optimization and design of such leads must take into account the requirements of the electrical and cryogenic systems as well as the local environment. The paper summarizes the results of the analysis made on HTS current leads, with special attention to the optimization process to be followed in different scenarios that include both DC and pulsed operation. Case studies are also presented and discussed. The calculation of the optimum performance in the various operating conditions was made assuming a lead design of the type used for LHC.
\end{abstract}

Index Terms-Accelerator technology, cryogenics, DC and pulsed powering, HTS current leads.

\section{INTRODUCTION}

$\mathbf{T}$ HE use of High Temperature Superconducting (HTS) current leads has become, in the last few years, a viable choice for the powering of superconducting magnet systems. Their potential for reducing the heat load to the cryogenic environment makes them attractive mainly for large scale superconducting accelerators, operating at liquid helium temperatures, but also for smaller isolated applications, e.g. cryo-cooled magnets operating at temperatures below $77 \mathrm{~K}$. In both cases, the derived savings contribute to relieve the load on the cryogenic system, freeing capacity for other use.

The optimization of self-cooled conventional current leads has been widely studied in the past. Analytical and numerical models for the calculation of the DC losses into the helium bath are available, and they all converge on a minimum value of about $1.1 \mathrm{~W} / \mathrm{kA}$ for a lead operating at nominal current between room temperature and $4.2 \mathrm{~K}$ [1]. The use of HTS material enables a saving of a factor of up to 10 [2].

The Large Hadron Collider (LHC) is equipped with more than a thousand HTS leads operating, in quasi-DC mode, at currents ranging from $600 \mathrm{~A}$ to $13000 \mathrm{~A}$ and transferring altogether more than $3 \mathrm{MA}$ to/from the liquid helium bath. The conceptual and detailed design of these leads has been

Manuscript received August 25, 2006.

The author is with the European Organization for Nuclear Research (CERN), 1211 Geneva 23, Switzerland (e-mail: amalia.ballarino@cern.ch).

Color versions of one or more of the figures in this paper are available online at http://ieeexplore.ieee.org.

Digital Object Identifier 10.1109/TASC.2007.898448 made at CERN, where pre-series units have been assembled and tested [3]. The extensive R\&D program carried out within this project, followed by the still ongoing construction and tests in cryogenic conditions of respectively 950 and 700 leads, increased confidence in the long-term and large-scale application of these devices. Even though in the last years much progress has been made to incorporate successfully HTS material in electrical components (e.g. power transmission cables, fault current limiters, small magnets, and insert coils), HTS current leads still today represent the only application that has reached the maturity needed for commercial industrialization.

The LHC HTS current leads incorporate HTS material in the form of stacks of Bi-2223 tape [4]. The design of these leads is such that, by cooling their upper-resistive part with $20 \mathrm{~K}$ helium gas, available at that temperature from the recovery line cooling the accelerator beam screen, the warm end of the HTS is maintained at $50 \mathrm{~K}$ [3]. These operating conditions are favorable for the HTS electrical performance, and represent an optimum in terms of exergetic cost of the refrigeration within the temperature levels of the LHC cryogenic system. Other systems, having available either helium gas at different temperatures or different cryogens, should be optimized taking into account their own boundary conditions.

This paper summarizes the results of the analysis performed on HTS current leads. Numerical simulations were carried out to optimize HTS leads operating in both DC and pulsed mode. The design of the LHC leads-type and geometry of resistive heat exchanger and of HTS element, scaled for different currents-has been used for the calculation of optimum performance in various operating conditions. Case studies are also presented and discussed.

\section{HTS LEADS IN DC MODE}

\section{A. Resistive Part}

Self-cooled conventional current leads have a minimum heat load into the helium bath of about $1.1 \mathrm{~W} / \mathrm{kA}$ [1]. This value, which is practically independent of the material properties, represents the optimum performance of a lead operating at nominal current. The correspondent geometry depends on the properties of the conductor and is characterized by an optimum shape factor $(S F)$, given by the product of the current $(I)$ times the length of the conductor $(L)$ divided by its cross section $\left(A_{C u}\right)$. These conclusions are based on a number of assumptions: equal temperatures of helium and conductor at the cold end of the lead, perfect heat exchange between the conductor and the gas, and the validity of the Wiedemann-Franz law that establishes a direct proportionality between the product of thermal conductivity times the electrical resistivity and the temperature. Furthermore, 
the helium mass flow is bound to the heat load by its latent heat of vaporization. These assumptions make it possible to use analytical models to calculate the optimum performance of a lead.

The cooling of the resistive part of the HTS lead can be made with cryogens at any temperature below the critical temperature of the HTS. In the case of helium, the whole range $5 \mathrm{~K}-85 \mathrm{~K}$ can be exploited and, for a given inlet temperature of the gas, a wide range of operating temperatures for the warm end of the HTS $\left(T_{H T S}\right)$ can be established. To investigate the impact of these parameters on the lead performance, the system of one-dimensional differential equations (1) expressing the heat balance between the conductor and the coolant was solved numerically:

$$
\begin{aligned}
\frac{d}{d x}\left(k_{C u}(T) A_{C u} \frac{d T}{d x}\right)+\frac{\rho(T) I^{2}}{A_{C u}}-P h(\vartheta)(T-\vartheta) & =0 \\
P h(\vartheta)(T-\vartheta)-\dot{m} c_{p} \frac{d \vartheta}{d x} & =0 .
\end{aligned}
$$

Thermal conduction in the helium gas has been neglected. The case of copper with residual resistivity ratio (RRR) of 70 has been analyzed. Conductor properties (thermal conductivity, $k_{C u}$, and electrical resistivity, $\rho$ ) were taken as temperature dependent, as was the heat transfer coefficient, $h$, between the lead and the gas. The latter, is obtained from the equation for laminar flow that considers the Nusselt number equal to a constant value (3.8 in this case). Input values are the hydraulic parameters of the cooling circuit (wetted perimeter, $P$, and equivalent diameter, $D_{e q}$ ). Boundary conditions are the bottom cold temperature of the heat exchanger $\left(T_{H T S}\right)$ and the inlet temperature of the gas $\left(T_{\mathrm{He}}\right)$. After normalization of the variable $x(u=x / L)$, the minimum helium mass flow $(\dot{m})$ and the corresponding lead geometry $(S F)$, together with the conductor $(T)$ and helium $(\vartheta)$ temperature profiles, are obtained from the solution of (1).

The results of the calculations are reported in Fig. 1, where the curves (a) and (b) represent respectively the calculated minimum flow rate and the corresponding lead shape factor for $T_{H e}$ varying from $5 \mathrm{~K}$ to $80 \mathrm{~K}$ and $T_{H T S}$ from $20 \mathrm{~K}$ to $85 \mathrm{~K}$. These curves give a complete map of the performance of helium gas cooled resistive heat exchangers optimized with different boundary conditions. The curves (a) in Fig. 1 depend slightly on the material properties, and are valid to within $10-20 \%$. For instance, the use of copper with $\mathrm{RRR}=150$ requires an increase of the flow rate by about $20 \%$ when $T_{H T S}<50 \mathrm{~K}$ and by about $10 \%$ in the other cases.

The impact on the $S F$ is more important: it requires an increase of $60 \%$ and $28 \%$ respectively. For copper with different $\mathrm{RRR}$, the difference in $k_{C u}$ becomes significant at temperatures below $50 \mathrm{~K}$, which explains the more marked variation in parameters at temperatures below this value. Fig. 2 shows the calculated temperature profiles of the heat exchangers of Fig. 1 for the case $T_{H e}=20 \mathrm{~K}$ and $T_{H T S}$ in the range $30 \mathrm{~K}-80 \mathrm{~K}$. As for the case of conventional self-cooled current leads, the optimum performance in the resistive part of the HTS leads is characterized by the zero slope of the temperature profile of the conductor at the warm end.

The LHC current leads were optimized for operating at nominal current with $T_{H T S}=50 \mathrm{~K}$ and $T_{H e}=20 \mathrm{~K}$. The measured
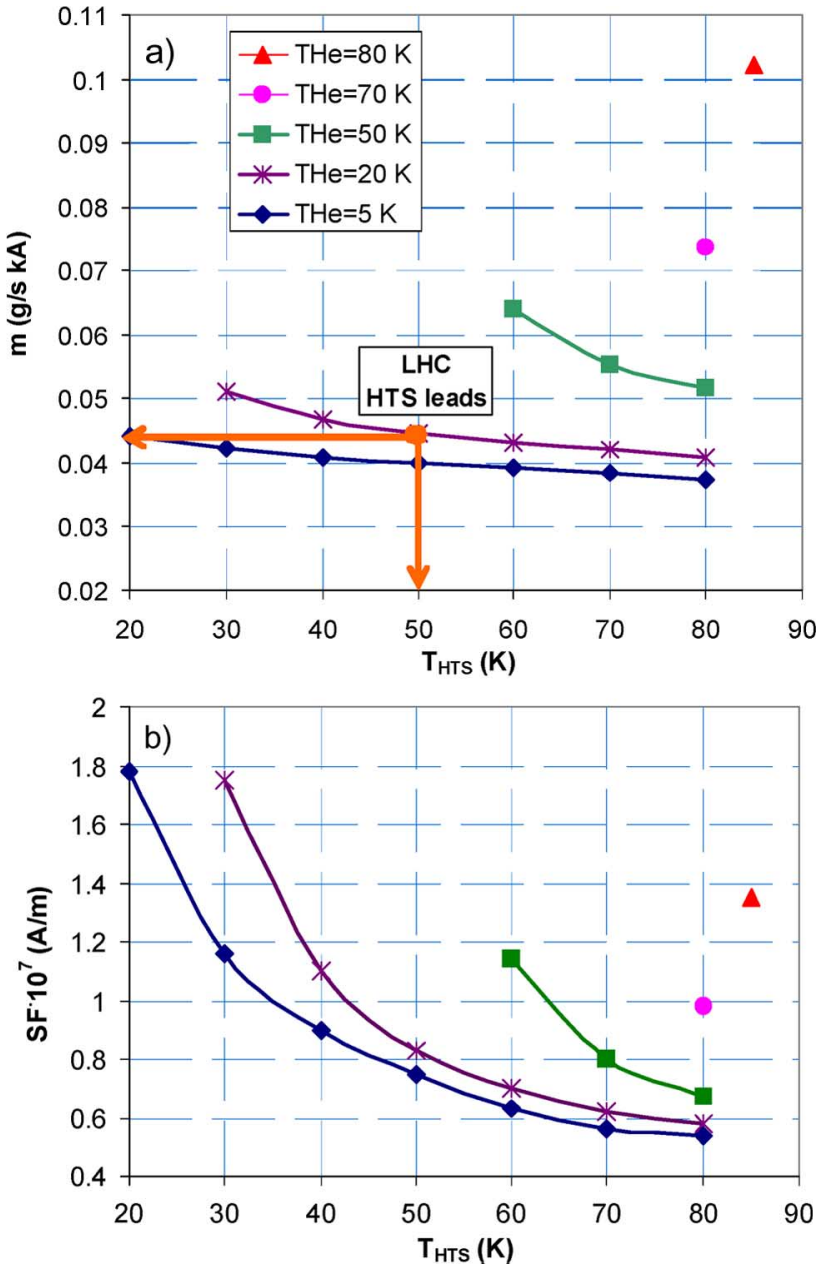

Fig. 1. Minimum helium mass flow rate (a, above) and shape factor ( $b$, below) for resistive part $(\mathrm{Cu}$ with RRR $=70)$ of HTS lead as a function of bottom temperature of the heat exchanger $\left(\mathrm{T}_{\mathrm{HTS}}\right)$ and inlet temperature of helium gas $\left(\mathrm{T}_{\mathrm{He}}\right)$.

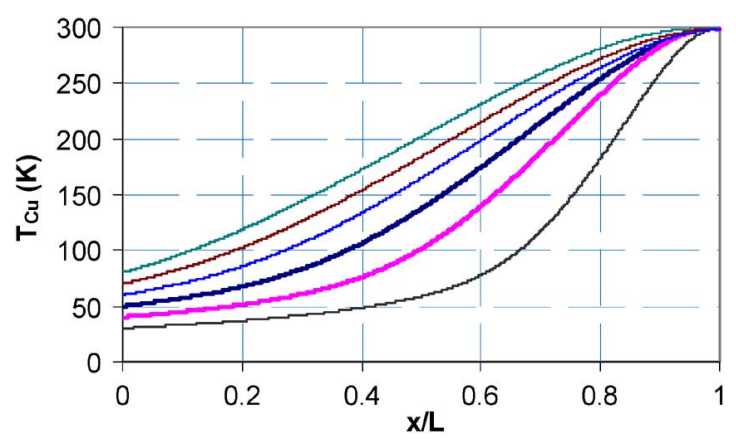

Fig. 2. Calculated temperature profile of resistive heat exchanger $(\mathrm{Cu}$ with $\mathrm{RRR}=70$ ) for $T_{H}=20 \mathrm{~K}$ and $\mathrm{T}_{\mathrm{HTS}}$ equal respectively to $30 \mathrm{~K}, 40 \mathrm{~K}$, $50 \mathrm{~K}, 60 \mathrm{~K}, 70 \mathrm{~K}$ and $80 \mathrm{~K}$ (from bottom to top).

mass flow rates are $0.6 \mathrm{~g} / \mathrm{s}$ for the 13000 A leads and $0.03 \mathrm{~g} / \mathrm{s}$ for the 600 A leads, in good agreement with the $0.045 \mathrm{~g} / \mathrm{s} \mathrm{kA}$ shown in Fig. 1. The 6000 A LHC HTS leads require a flow of $0.33 \mathrm{~g} / \mathrm{s}$. The $2 \%$ increase from the ideal flow rate was expected in view of the requirement of being able to run these leads in DC mode with currents of up to $7500 \mathrm{~A}$. 
In the case of cooling with a nitrogen bath, the optimization process for the heat exchanger is the same as for conventional self-cooled leads. The losses at $77 \mathrm{~K}$ are about $23 \mathrm{~W} / \mathrm{kA}$.

\section{B. HTS Part}

The HTS part of the LHC current leads is made of stacks of $\mathrm{Bi}-2223$ tapes with a gold-doped silver matrix, where the gold percentage is about $5 \%$ in weight [4]. The use of stacks in leads, which is convenient in view of their robustness, is possible thanks to the possibility of using straight conductors. The stacks have a constant cross section. Their critical current $\left(I_{c}\right)$ depends on the characteristics and number $(n)$ of tapes, and shows a typical reduction of $30 \%$ with respect to the value calculated by multiplying $n$ times the $I_{c}$ of each tape [4]. The geometrical disposition of the stacks in the HTS unit is such as to minimize the perpendicular component of the self-field.

The HTS unit operates in a range of temperature from $T_{H T S}$ to $4.2 \mathrm{~K}$ either in self-cooling, as for the LHC leads, or in conduction cooling conditions. Convection cooling due to gas boil-off improves the thermal performance by a factor of up to 5 with respect to the conduction cooled option.

The total heat load into the helium bath depends on $T_{H T S}$, on the number of tapes needed for transporting the current and on the requirements of the electrical circuit with regard to the protection of the HTS part. A reduction by a factor of 10 with respect to conventional self-cooled leads has been calculated and measured on the 13000 A LHC HTS leads [2], [3]. The total reduction in cooling power is a factor of 3 .

\section{HTS LEADS IN PULSED MOdE}

\section{A. Resistive Part}

HTS leads dimensioned for operating in DC mode at the maximum current can be used to power pulsed superconducting magnets. However, if the superconducting circuit never operates in DC conditions, the leads will be constantly over-cooled, and it will always be necessary to apply heat at their warm end for maintaining the room temperature. Otherwise, humidity and ice will build up, compromising the electrical insulation of the circuit.

The analysis of leads operating in AC mode has been made by solving numerically the system of non-linear second order partial differential equations (2) expressing the heat balance between the lead and the gas in transient conditions:

$$
\begin{aligned}
c_{C u}(T) \nu_{C u} A_{C u} \frac{\partial T}{\partial t}= & \frac{\partial}{\partial x}\left(k_{C u}(T) A_{C u} \frac{\partial T}{\partial x}\right) \\
& +\frac{\rho(T) I(t)^{2}}{A_{C u}}+-P h(\vartheta)(T-\vartheta) \\
c_{H e} \nu_{H e}(\vartheta) A_{f} \frac{\partial \vartheta}{\partial t}= & P h(\vartheta)(T-\vartheta)-\dot{m} c_{p} \frac{\partial \vartheta}{\partial x} .
\end{aligned}
$$

The algorithm adopted for solving (2) is the method of lines, a semi-discrete method that uses finite difference relationships for the spatial $(x)$ derivatives and ordinary differential equations

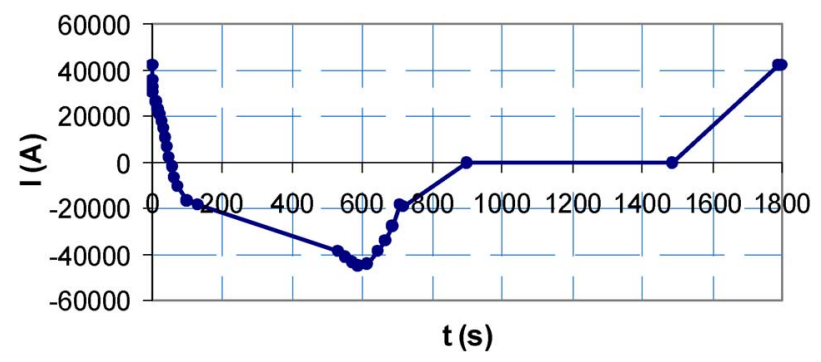

Fig. 3. Cycle of the ITER poloidal coils $\left(t=1800 \mathrm{~s}, I_{M A X}=45.4 \mathrm{kA}\right)$.

for the time $(t)$ derivatives. As in (1), material and helium gas properties are temperature dependent. $c_{C u}, \nu_{\mathrm{Cu}}, \nu_{\mathrm{He}}$ and $A_{f}$ are respectively the specific heat and the density of the copper, the density and the cross section of the helium. Boundary conditions are the temperatures $T_{H e}(\vartheta(x=0, t))$ and $T_{H T S}(T(x=$ $0, t)$ ). Initial conditions are the temperature profiles of helium $(\vartheta(x, t=0))$ and conductor $(T(x, t=0))$.

Several cases have been studied. For those discussed here the boundary conditions are $T_{H e}=20 \mathrm{~K}$ and $T_{H T S}=50 \mathrm{~K}$.

Slow-Cycle: The ITER PF coils. The ramp rate of the six ITER poloidal coils (period of $1800 \mathrm{~s}$ ) has been taken from an ITER reference operation scenario (Fig. 3) [5].

A copper heat exchanger with $\mathrm{RRR}=70$ and optimized for operating in DC mode $\left(A_{C u}=A_{D C}\right)$ at the maximum current $\left(I_{M A X}=45400 \mathrm{~A}\right)$ requires a flow of $2.2 \mathrm{~g} / \mathrm{s}$. The same heat exchanger carries the current I(t) of Fig. 3 with a flow of $1.6 \mathrm{~g} / \mathrm{s}$.

If the conductor cross-section is increased by $20 \%$ in the top half of the upper part $(L=0.5 \mathrm{~m})$, the lead operates in the same temperature range with a flow of $1 \mathrm{~g} / \mathrm{s}$. No overheating above room temperature occurs, thanks to the increased cross section in the warmer region, and the peak voltage is $75 \mathrm{mV}$ when the current is $\left|I_{M A X}\right|$. The highest temperatures of each cycle occur in the time interval $590 \mathrm{~s}-700 \mathrm{~s}$, when the current increases up to $I_{M A X}$ with the highest ramp rate of $113 \mathrm{~A} / \mathrm{s}$. The calculated voltage drop $(U)$ and temperature profiles are shown in Fig. 4.

Fast-Cycle: Dipoles for the SPS upgrade and for the FAIR project.

The need of fast pulsed superconducting magnets (1 T/s) for the SPS injector is being studied in the framework of future LHC upgrades: pulsed repetition rates of $12 \mathrm{~s}$ bring the current from injection (500 A) to $10000 \mathrm{~A}$ (Fig. 5) [6].

Fast ramp rates will be used for the powering of the superconducting dipoles (4 T/s) of the GSI FAIR project, where pulsed triangular cycles of $1 \mathrm{~Hz}$ frequency and $I_{M A X}=6000 \mathrm{~A}$ have been used for these simulations [7].

For the SPS leads, the use of a variable cross section, with a $30 \%$ reduction of the $A_{C u}$ value in the bottom-half part, enables a reduction of the flow from $0.3 \mathrm{~g} / \mathrm{s}$ to $0.25 \mathrm{~g} / \mathrm{s}$. After about 20 minutes of run, the temperature profile reaches a practically zero slope at room temperature with temperature variations of less than $1.5 \mathrm{~K}$ during a cycle (Fig. 5).

Also for the GSI leads, the increase of the cross-section in the top half gives a reduction in the nominal flow rate to $0.14 \mathrm{~g} / \mathrm{s}$. The maximum voltage during a cycle is $89 \mathrm{mV}$ after $0.5 \mathrm{~s}$, and the temperature variations within one cycle are less than $1 \mathrm{~K}$. 


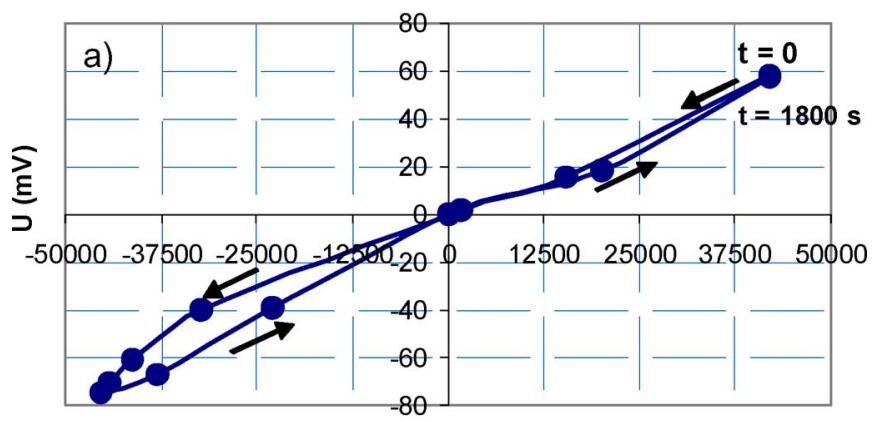

I (A)

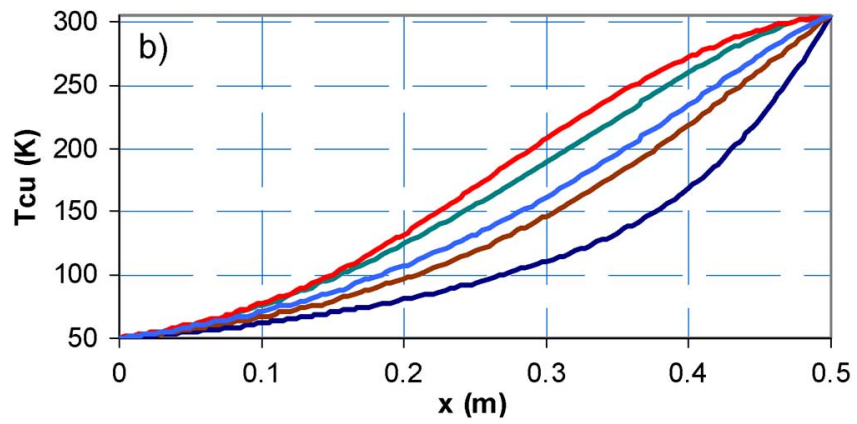

Fig. 4. Calculated voltage drop (a, above) and temperature profile (b, below, at $\mathrm{t}=50 \mathrm{~s}, 500 \mathrm{~s}, 1800 \mathrm{~s}, 580 \mathrm{~s}$ and $700 \mathrm{~s}$ from bottom to top) of heat exchanger carrying the current I(t) of Fig. 2 and with variable cross section.
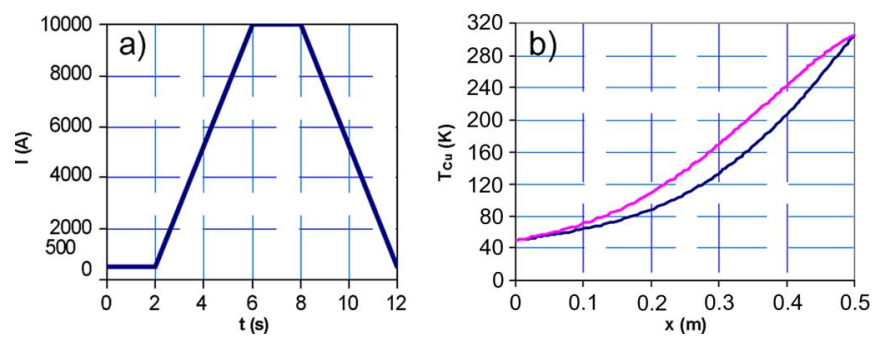

Fig. 5. Current cycle (a, left) and temperature profile (b, right) of the SPS leads in the case of variable (b, top curve) and constant ( $b$, bottom curve) cross sections $(\mathrm{t}=20$ minutes $)$.

\section{B. HTS Part}

The AC losses of the superconductor have to be added to the thermal conduction losses. Hysteresis losses in the superconductor can be calculated by simplifying the superconducting geometry of the Bi-2223 tapes in the stack with a single core ellipse and by applying the Norris formula [8]. This assumption is in good agreement with losses measured on LHC stacks [9].

In the leads, the HTS operates at fixed frequency and fixed transport current, and the normalized current $\left(I_{M A X} / I_{C}\right)$ decreases from $T_{H T S}$ down to the cold end of the superconductor $\left(I_{M A X} / I_{c}(T(x))\right.$. The advantage of operating along the stack's length at currents well below the critical one- together with the very low frequencies of the cases of interest and the relatively short length of the superconductor ( $L$ is usually $\leq 0.5 \mathrm{~m}$ ) - reduces the hysteresis losses to a small fraction of the thermal conduction value. For instance, the hysteresis loss of an HTS element for the GSI lead of Section III $(L=0.4 \mathrm{~m})$, operating at $I_{M A X}=6000 \mathrm{~A}$ with $T_{H T S}=77 \mathrm{~K}$ and a current equal to $90 \%$ of its critical value at that temperature, is about $12 \mathrm{~mW}$.

For the eddy current losses in the silver-gold matrix, the dependence of the resistivity on the temperature-it decreases by a factor of about 2.5 from $77 \mathrm{~K}$ down to $4.2 \mathrm{~K}$-would mean they have some impact at higher frequencies (about $100 \mathrm{~mW}$ for the GSI HTS module operating at $50 \mathrm{~Hz}$ ). At the low frequencies of interest for this study, they represent a negligible fraction of the total losses.

\section{CONCLUSION}

The choice of the operating conditions of a HTS lead should be done within the framework of the cryogenic and electrical system where the lead will operate. The operating conditions have an impact on the optimization process and on the global exergetic cost of the refrigeration.

The principles of optimization of DC and pulsed HTS current leads have been discussed. A guide has been presented for optimized performance with different boundary conditions in DC mode. For pulsed operation, case studies have been discussed and the benefit of a variable cross-section in the resistive part has been demonstrated. The design of the LHC HTS leads has been used as the basis for all calculations presented. The incorporation of a resistive heat exchanger with non-uniform cross-section would not complicate the manufacture and assembly of leads of this design.

\section{REFERENCES}

[1] M. Wilson, Superconducting Magnets. Oxford: Clarendon Press, 1983, pp. 256-278.

[2] A. Ballarino, "HTS current leads for the LHC magnet powering system," Physica C, vol. 372-376, pp. 1413-1418, 2002.

[3] A. Ballarino, S. Mathot, and D. Milani, "13000 A HTS current leads for the LHC accelerator: From conceptual design to prototype validation," in Proc. of Eucas 2003, Sorrento, Italy, LHC Project Report 696.

[4] A. Ballarino, L. Martini, S. Mathot, T. Taylor, and R. Brambilla, "Large scale assembly and characterization of Bi-2223 HTS conductors," in Proceedings of ASC 2006, Seattle, 2006.

[5] ITER Cryoplant Interface Meeting. CEA, Grenoble, 2005.

[6] W. Scandale, "LHC-Upgrade based on a novel high intensity high energy injector chain," in CARE Workshop, 2005 [Online]. Available: http://care-hhh.web.cern.ch/care\%2Dhhh/publications.htm

[7] A. Kovalenko et al., "Design and study of superferritic model dipole and quadrupole magnets for the GSI fast-pulsed synchrotron SIS100," in Proc. of EPAC 2004, Lucerne, Switzerland.

[8] W. T. Norris, "Calculation of hysteresis losses in hard superconductors carrying ac: Isolated conductors and edges of thin sheets," Journal of Physics D: Appl. Phys., vol. 3, pp. 489-507, 1970.

[9] S. Ginocchio, A. Ballarino, E. Perini, and S. Zanella, "DC and AC electrical characterization of stacks of HTS tapes," in Proceedings of ASC 2006, Seattle, 2006. 